\title{
Gingival crevicular fluid calprotectin, osteocalcin and cross-linked N-terminal telopeptid levels in health and different periodontal diseases
}

\author{
Sema Becerik ${ }^{\mathrm{a}}$, Beral Afacan ${ }^{\mathrm{a}}$, Veli Özgen Öztürk ${ }^{\mathrm{a}}$, Harika Atmaca ${ }^{\mathrm{b}}$ and Gülnur Emingil ${ }^{\mathrm{a}, *}$ \\ ${ }^{a}$ Ege University, School of Dentistry, Department of Periodontology, Izmir, Turkey \\ ${ }^{\mathrm{b}}$ Celal Bayar University, School of Science, Department of Biology, Manisa, Turkey
}

\begin{abstract}
Aim: The aim of the present study was to investigate gingival crevicular fluid (GCF) calprotectin, osteocalcin and cross-linked N-terminal telopeptide (NTx) levels in health along with different periodontal diseases.

Material and methods: Twenty chronic periodontitis (CP), 20 generalized aggressive periodontitis (G-AgP), 20 gingivitis and 20 healthy subjects were included. Probing depth, clinical attachment level, plaque index and papillary bleeding index was recorded. GCF calprotectin, osteocalcin and NTx levels were analyzed by enzyme-linked immunosorbent assay (ELISA).

Results: CP, G-AgP and gingivitis groups had higher GCF calprotectin total amount compared to healthy subjects $(p<0.008)$. $\mathrm{CP}$ and G-AgP groups had similar, but higher levels compared to gingivitis groups $(p<0.008)$. CP and G-AgP groups had lower GCF osteocalcin total amount compared to gingivitis and healthy groups $(p<0.008)$. CP group had higher GCF NTx but lower osteocalcin total amount and osteocalcin/NTx ratio than the G-AgP group $(p<0.008)$.

Conclusions: Our results suggest that elevated GCF calprotectin levels play a role as a reliable inflammatory marker in the pathogenesis of periodontal disease. Fluctuating GCF levels of osteocalcin and NTx might point out to the abnormal bone turnover in periodontitis. Our data document for the first time the role of NTx in the pathogenesis of different periodontal diseases.
\end{abstract}

Keywords: Calprotectin, osteocalcin and NTx in periodontitis

\section{Introduction}

Current knowledge about the pathogenesis of periodontal disease suggests that the central cause of periodontal disease is the loss of a healthy balance between microbial virulence agents and host inflammatory response $[1,2]$. The immune system while protecting the host against microbial dental plaque, also participates in attacking the host. Inflammation and tissue destruction are early and continuing events during host- mediated process in response to the bacterial infection [3].

* Corresponding author: Gülnur Emingil, DDS, PhD, Ege University, School of Dentistry, Department of Periodontology, Bornova35100, Izmir-Turkey. Tel.: +90 232388 1105; Fax: +90 232388 0325; E-mail: gemingil@yahoo.com.
Periodontal diseases may differ in their etiological factors and pattern of progression. This variability can be attributed to differences in the presence of factors that might modify the host response to microbial pathogens. Chronic periodontitis (CP) and aggressive periodontitis, two forms of inflammatory periodontal disease, differ from each other in terms of the magnitude, sequel and control of the response [4].

The destruction of soft and hard tissues seen in periodontitis is caused by a large number of cytokines as well as due to the presence of other effector molecules released by resident and migrating cells [2,5]. Calprotectin is a $36.5-\mathrm{kDa}$ calcium and zinc binding protein consisting of one light (8 $\mathrm{kDa}, \mathrm{MRP} 8)$ and one heavy (14 $\mathrm{kDa}, \mathrm{MRP} 14)$ subunit that belong to the S100 protein family [6]. Calprotectin is constitutively expressed in the cytosol of neutrophils, monocytes, 
activated macrophages, and keratinocytes and released during activation or death of these cells. Calprotectin complex, also known as L1 antigen, calgranulin A and $\mathrm{B}$, macrophage migration inhibitory factor-related protein 8 and 14 (MRP8 and MRP14), S100A8/S100A9, and cystic fibrosis antigen, has several functions in inflammatory reactions [7]. It acts as a chemotactic factor and regulates adhesion and migration of neutrophils or monocytes and is therefore considered a pro-inflammatory marker [6]. It also shows in vitro antimicrobial and antifungal activities against the periodontopathic bacteria as well as Candida strains [8, 9]. It has been previously shown that calprotectin was present in human dental calculus and suggested that it might be derived from gingival crevicular fluid (GCF), saliva and dental plaque [10]. Studies have shown that calprotectin level in GCF from periodontitis patients is higher than that from healthy subjects and positively correlated with clinical and biochemical markers of periodontal inflammation [11-14].

Bone homeostasis maintains by a coupled process of resorption followed by formation which reflect a change in bone turnover [15]. Markers of bone formation are proteins revealing osteoblast activity and are byproducts of collagen synthesis, matrix proteins or osteoblastic enzymes [16,17]. Osteocalcin is a small $(5.4 \mathrm{kDa})$, calcium-binding protein of bone accounting for $10-20 \%$ of the non-collagenous protein in bone matrix. It has three residues of a calcium-binding amino acid, gamma-carboxyglutamic acid (Gla), that allow specific conformational changes enabling its binding to hydroxyapatite and later accumulation in bone matrix $[18,19]$. This vitamin K- and D-dependent protein produced by mature osteoblasts, osteocytes and odontoblasts, is found in the extracellular mineralized matrix of bone and in the serum of circulating blood [18, 20]. It may be involved in regulation of osteoblast function, regulation of bone turnover and/or mineralization. Markers of bone resorption, which reflect osteoclastic activity are mostly the breakdown products of type I bone collagen, the main component of the organic bone matrix $[16,17]$. N-terminal cross-linked telopeptide of type I collagen (NTx) is the aminoterminal peptides of mature type I collagen with the cross-links attached and is released during bone resorption $[21,22]$. The NTx molecule, a reliable marker for subtle changes in bone turnover, can be measured in either serum or urine and used as a marker of bone resorption in several systemic diseases [23,24].

It is well known that abnormal activation of the immune system leads to bone destruction in periodon- tal diseases. The increased recognition of the interactions between the cells of the immune and skeletal system has led to the seeking of a variety of regulatory molecules that play a role in the progression of periodontal disease [25-27]. In previous studies, the bestknown markers of the bone turnover have been widely investigated in GCF samples of patients with different periodontal diseases and implicated to be associated with the progression of periodontal diseases [28-30]. Several investigations provided contradictory evidence about the role of osteocalcin in GCF of patients with periodontitis [31-33]. Little is known about the role and presence of the novel NTx in periodontal diseases. Moreover, there is lack of information about the GCF calprotectin, osteocalcin and NTx levels of patients with generalized aggressive periodontitis (G-AgP) and $\mathrm{CP}$ and gingivitis. Therefore, the aim of the present study was to examine how the GCF calprotectin, osteocalcin and NTx levels changes in the presence of gingival health, gingival inflammation (in gingivitis) and/or periodontal tissue destruction (in $\mathrm{G}-\mathrm{AgP}$ and $\mathrm{CP}$ ) and to test whether calprotectin, osteocalcin and NTx levels are correlated with clinical parameters.

\section{Materials and methods}

\subsection{Study population}

A total of 80 subjects were included in this study. All consecutive subjects were recruited from the Department of Periodontology, School of Dentistry, Ege University, Izmir, Turkey. The purpose and procedures were explained to all subjects prior to participation, and all participants gave written informed consent in accordance with Helsinki declaration. The study protocol was approved by the Ethics Committee of the Ege University School of Medicine. The purpose of the study was completely explained to each subject before entering the study and informed consent was obtained from each subject. Complete medical and dental histories were taken from all subjects. All of the patients were non-smokers. None of the subjects had a history of systemic disease and had received antibiotics or other medications or periodontal treatment within the past 4 months. Patients with severe medical disorders including diabetes mellitus and immunological disorders as well as alcoholics were excluded from the study. Post-menopausal, pregnant and lactating females and those taking oral contraceptive drugs were also excluded from the study. The selection of the patients was 
made according to the clinical and radiographic criteria proposed by the 1999 International World Workshop for a Classification of Periodontal Disease and Conditions [34].

\subsubsection{Generalized aggressive periodontitis group $(G-A g P)$}

The G-AgP group included 11 females and 9 males ranged in age from 19 and 39 with a mean age of $31.4 \pm$ 6.8 years. These patients demonstrated a generalized pattern of severe destruction and clinical attachment loss (CAL) of $\geqslant 5 \mathrm{~mm}$ and probing depth (PD) $\geqslant 6 \mathrm{~mm}$ on 8 or more teeth; at least 3 of those were other than central incisors or first molars. Additionally, CAL was not consistent with the amount of plaque accumulation or local contributing factors. All subjects had at least 16 teeth.

\subsubsection{Chronic periodontitis group $(C P)$}

The CP group consisted of 9 females and 11 males between the ages of 35 to 50 (mean of $43.1 \pm 4.2$ years). They had moderate to severe alveolar bone loss and CAL of $\geqslant 5 \mathrm{~mm}$ and PD of $\geqslant 6 \mathrm{~mm}$ in multiple sites of all four quadrants of the mouth, but with no evidence of rapid progression. Diagnosis of $\mathrm{CP}$ was made if the CAL was commensurate with the amount of plaque accumulation of the patients and all had at least 16 teeth in their mouth.

\subsubsection{Gingivitis group}

The gingivitis group, ranged in age from 22 to 54 (mean age $38.0 \pm 9.7$ years), included 8 females and 12 males. They had varying degrees of gingival inflammation, but no CAL $>2 \mathrm{~mm}$, no sites with alveolar bone loss present in radiography (i.e., distance between the cemento-enamel junction and bone crest at $>95 \%$ of the proximal tooth sites $\leqslant 3 \mathrm{~mm}$ ).

\subsubsection{Healthy group}

The healthy group consisted of 11 females and 9 males who exhibited PD $<3 \mathrm{~mm}$ and no CAL, clinical inflammation and sulcular bleeding (mean age $43.6 \pm$ 9.9 years; range 30 to 61 years). These individuals were healthy volunteers from the Department of Periodontology. No radiographic evidence of alveolar bone loss was observed in these patients (i.e., distance between the cemento-enamel junction and bone crest $\leqslant 3 \mathrm{~mm}$ at $>95 \%$ of the proximal tooth sites).

\subsection{Determination of periodontal status}

At the screening stage, to determine the clinical periodontal status, all subjects had a clinical periodontal examination including PD, CAL, papilla bleeding index (PBI) [35] and plaque index (PI) [36] by one examiner (S.B). PD measurements were performed using manual Williams probe. All measurements were performed at 6 sites per tooth for whole mouth.

\subsection{Collection of GCF samples}

After being selected for the study, subjects were recalled for GCF sampling. In the G-AgP and CP groups, GCF samples were collected from two approximal sites of anterior teeth with $\geqslant 6 \mathrm{~mm}$ PD. In the gingivitis group, GCF sampling was done from two approximal sites of anterior teeth with bleeding on probing and PD $\geqslant 2 \mathrm{~mm}$. In the healthy group, GCF samples were collected from two approximal sites of two teeth with $\mathrm{PD} \leqslant 2 \mathrm{~mm}$. Prior to GCF sampling, the supragingival plaque was removed from the interproximal surfaces with a sterile curette; these surfaces were dried gently by an air syringe and were isolated by cotton rolls. GCF was sampled with filter paper (Periopaper, ProFlow, Inc., Amityville, NY, USA). Paper strips were carefully inserted into the crevice until mild resistance was felt and left there for 30 seconds [37]. Care was taken to avoid mechanical injury. Strips contaminated with blood were discarded [38]. The absorbed GCF volume of each strip was determined by electronic impedance (Periotron 8000, ProFlow, Inc., and Amityville, NY, USA), pooled and placed into a sterile eppendorff vials and kept at $-40^{\circ} \mathrm{C}$ until being analyzed. The readings from the Periotron 8000 were converted to an actual volume $(\mu \mathrm{l})$ by reference to the standard curve.

\subsection{Analysis of calprotectin, osteocalcin and NTX}

GCF samples were eluted from the strips by placing them in $300 \mu \mathrm{l}$ of PBS. GCF calprotectin (Immundiagnostik AG, Bensheim, Germany), osteocalcin (Bender Med Systems, Vienna, Austria) and NTx (Wampole Laboratories, Princeton, NJ, USA) levels were assayed by using commercially available ELISA kits. Procedures were performed according to the instructions in the kit. The minimum detectable limits of calprotectin, osteocalcin and NTx were $1.9 \mathrm{pg} / \mathrm{ml}, 0.2 \mathrm{ng} / \mathrm{ml}$ and $3.2 \mathrm{nM}$ bone collagen equivalent (BCE), respectively. The amounts of calprotectin, osteocalcin and NTx in each sample were calculated based on the dilutions 
Table 1

Clinical parameters of the sampling areas in study groups (mean \pm SD)

\begin{tabular}{lcrcc}
\hline & G-AgP & \multicolumn{1}{c}{ CP } & Gingivitis & Healthy \\
\hline PD $(\mathrm{mm})$ & $5.8 \pm 0.8^{\ddagger \dagger}$ & $7.2 \pm 0.5^{\ddagger}$ & $2.5 \pm 0.5^{*}$ & $1.5 \pm 0.4$ \\
CAL $(\mathrm{mm})$ & $6.8 \pm 1.5^{\ddagger \dagger}$ & $8.2 \pm 1.2^{\ddagger}$ & - & - \\
PBI & $2.2 \pm 1.1^{*}$ & $3.1 \pm 0.5^{\ddagger}$ & $2.2 \pm 0.9^{*}$ & $0.35 \pm 0.4$ \\
PI & $3.9 \pm 0.9^{*}$ & $4.2 \pm 0.5^{*}$ & $3.75 \pm 0.8^{*}$ & $1.25 \pm 0.5$ \\
GCF $(\mu \mathrm{l})$ & $0.76 \pm 0.2^{*}$ & $0.77 \pm 0.1^{\ddagger}$ & $0.60 \pm 0.2^{*}$ & $0.19 \pm 0.1$ \\
\hline
\end{tabular}

PD: Probing depth, CAL: Clinical attachment loss, PBI: Papillary bleeding index, PI: Plaque index, GCF: Gingival crevicular fluid, G-AgP: Generalized aggressive periodontitis, CP: Chronic periodontitis.

${ }^{*}$ Significant difference from healthy group (Kruskal Wallis test, $p<0.05$, Mann-Whitney U test, $p<0.008$ ).

${ }^{\dagger}$ Significant difference from chronic periodontitis group (Kruskal Wallis test, $p<0.05$, Mann-Whitney U test, $p<0.008$ ).

${ }^{\ddagger}$ Significant difference from gingivitis and healthy groups (Kruskal Wallis test, $p<0.05$, Mann-Whitney U test, $p<0.008$ ).

and the results were expressed as total amount in the 30 second of the two GCF sample. Calculation of the concentration data for each mediator was performed by dividing the amount of each mediator by the GCF volume.

\subsection{Statistical analysis}

Considering a difference of $50 \%$ in mean GCF levels of the biochemical markers and assuming standard deviations to be maximum $80 \%$ of the mean values and accepting a power of $90 \%$, P-value of $5 \%$ in healthy and diseased groups, minimum sample size was calculated. Power calculation analysis revealed that the minimum required sample size was nine subjects for each group. Statistical analysis was performed using nonparametrical techniques. Comparisons between the study groups were performed using the Kruskal-Wallis test. When there were significant differences $(p<$ $0.05)$, post-hoc 2-group comparisons were assessed with Bonferroni-corrected Mann-Whitney U tests, and P-values $<0.008$ were considered to be statistically significant. Spearman rank correlation analysis was used to analyze the correlations between GCF calprotectin, osteocalcin and NTx levels and clinical parameters and $p<0.05$ was considered as significant. All data analysis was performed using a statistical package Abacus Concepts, Inc., Berkeley, CA, USA.

\section{Results}

\subsection{Clinical findings}

The mean clinical data for the sampling areas are shown in Table 1.

\subsubsection{PD and $C A L$}

The mean PD scores of sampling sites in G-AgP, CP and gingivitis groups were significantly higher than the healthy group $(p<0.008)$. CP group had higher scores compared to $\mathrm{G}-\mathrm{AgP}$ group and both groups had higher PD scores than the gingivitis group $(p<0.008)$. The mean CAL of sampling sites in G-AgP and CP groups were significantly higher than that of the gingivitis and healthy group $(p<0.008)$. CP groups had elevated CAL scores compared to G-AgP group $(p<0.008)$.

\subsubsection{PBI and PI scores}

All patient groups had significantly higher PBI and PI scores compared to the healthy group $(p<0.008)$. CP group had significantly elevated PBI scores compared to the gingivitis group $(p<0.008)$. G-AgP and gingivitis groups had similar PBI and PI scores $(p>$ 0.008).

\subsubsection{GCF scores}

All patient groups had significantly higher GCF scores compared to the healthy group $(p<0.008)$. CP group had significantly elevated GCF scores compared to the gingivitis group $(p<0.008)$. $\mathrm{G}-\mathrm{AgP}$ and gingivitis groups had similar GCF scores $(p>0.008)$.

\subsection{Biochemical findings}

\subsubsection{GCF calprotectin levels}

Distribution of the total amount of GCF calprotectin is shown in Fig. 1. Significant differences were found between study groups $(p=0.008)$. CP and $\mathrm{G}-\mathrm{AgP}$ groups had higher GCF calprotectin total amount compared to gingivitis and healthy groups. GCF calprotectin total amount of G-AgP group was similar to that of CP group $(p>0.008)$. Gingivitis group had signif- 


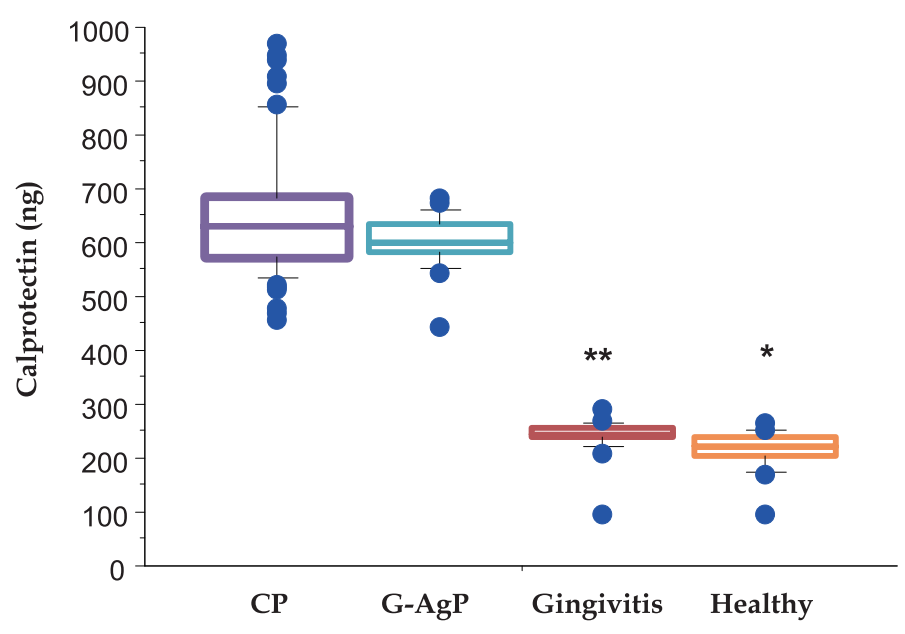

Fig. 1. GCF calprotectin levels (sample pooled from two sites) of CP, G-AgP, gingivitis and healthy groups. Box plots show medians, 25th and 75th percentiles as boxes, 10th and 90th percentiles as whiskers. Outside values are shown as blue circles. * Significantly different from G-AgP, $\mathrm{CP}$, and gingivitis groups. (Kruskal Wallis test, $p<0.05$, Mann-Whitney $\mathrm{U}$ test, $p<0.008$ ). ${ }^{* *}$ Significantly different from $\mathrm{G}-\mathrm{AgP}$ and $\mathrm{CP}$ groups. (Kruskal Wallis test, $p<0.05$, Mann-Whitney U test, $p<0.008$ ).

icantly higher GCF calprotectin total amount than the healthy group $(p<0.008)$ (Fig. 1).

When the data were expressed as concentration, patient groups had lower GCF calprotectin concentration compared to the healthy group $(p<0.008)$. Gingivitis group had also lower GCF calprotectin concentration than the $\mathrm{CP}$ and $\mathrm{G}-\mathrm{AgP}$ group $(p<0.008)$, while both $\mathrm{CP}$ and G-AgP groups had similar GCF calprotectin concentration $(p>0.008)$ (data not shown).

\subsubsection{GCF osteocalcin levels}

$\mathrm{CP}$ and G-AgP groups had lower GCF osteocalcin total amount compared to gingivitis and healthy groups. GCF osteocalcin total amount of CP group was significantly lower compared to those of G-AgP group ( $p<$ $0.008)$. Gingivitis group had similar GCF osteocalcin total amount to the healthy group $(p>0.008)$ (Fig. 2).

When the data were expressed as concentration, patient groups had lower GCF osteocalcin concentration compared to the healthy group $(p<0.008)$. Gingivitis group had higher GCF osteocalcin concentration than the $\mathrm{CP}$ and G-AgP group $(p<0.008)$, while both $\mathrm{CP}$ and G-AgP groups had similar GCF osteocalcin concentration $(p>0.008)$ (data not shown).

\subsubsection{GCF NTx levels}

CP and G-AgP groups had similar GCF NTx total amount to gingivitis and healthy groups $(p>0.008)$. G-AgP group had significantly lower GCF NTx total amount than the $\mathrm{CP}$ group $(p<0.008)$. Gingivitis group had similar GCF NTx total amount to the healthy group $(p>0.008)$ (Fig. 3).
When the data were expressed as concentration, patient groups had lower GCF NTx concentration compared to the healthy group $(p<0.008)$. Gingivitis group had higher GCF osteocalcin concentration than the $\mathrm{CP}$ and $\mathrm{G}-\mathrm{AgP}$ group $(p<0.008)$. G-AgP group had lower GCF NTx concentration compared to CP group ( $p<0.008$ ) (data not shown).

\subsubsection{GCF osteocalcin/NTx ratio}

Among the study groups CP group had the lowest osteocalcin/NTx ratio compared to the other groups $(p<0.008)$. G-AgP, gingivitis and healthy groups had similar GCF osteocalcin/NTx ratio $(p>0.008)$ (Fig. 4).

The correlation between GCF calprotectin, osteocalcin, NTx and osteocalcin/NTx ratio and clinical parameters of $\mathrm{G}-\mathrm{AgP}, \mathrm{CP}$, gingivitis and healthy groups is presented in Table 2. All clinical parameters were positively correlated with GCF calprotectin total amount $(p<0.05)$. GCF osteocalcin total amount was negatively correlated with all clinical parameters $(p<$ 0.05). There was no correlation between GCF NTx total amount and clinical parameters $(p>0.05)$. GCF osteocalcin/NTx ratio was negatively correlated with all clinical periodontal parameters $(p<0.05)$.

\section{Discussion}

In the present study, we investigated calprotectin, osteocalcin and NTx levels in GCF of patients with different periodontal diseases. The result of the present 


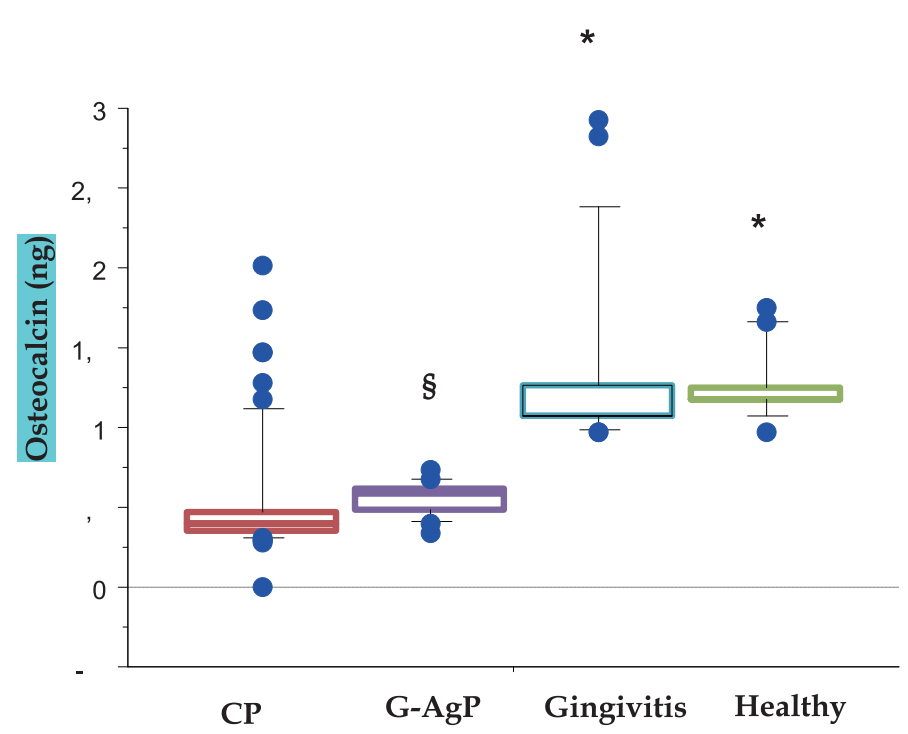

Fig. 2. GCF osteocalcin levels (sample pooled from two sites) of CP, G-AgP, gingivitis and healthy groups. Box plots show medians, 25th and 75th percentiles as boxes, 10 th and 90 th percentiles as whiskers. Outside values are shown as blue circles. ${ }^{*}$ Significantly different from G-AgP and CP groups. (Kruskal Wallis test, $p<0.05$, Mann-Whitney $\mathrm{U}$ test, $p<0.008$ ). ${ }^{\S}$ Significantly different from CP group. (Kruskal Wallis test, $p<0.05$, Mann-Whitney $\mathrm{U}$ test, $p<0.008$ ).

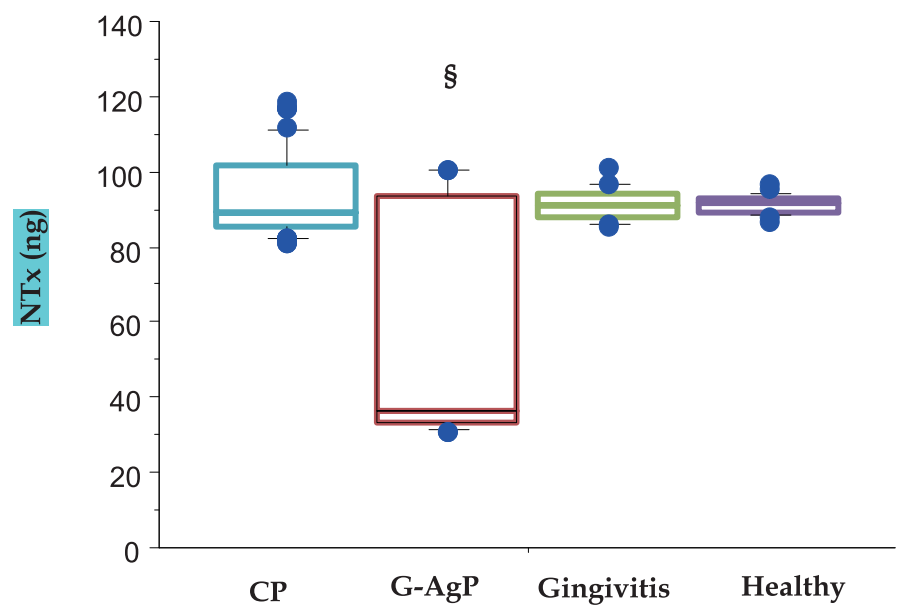

Fig. 3. GCF NTx levels (sample pooled from two sites) of CP, G-AgP, gingivitis and healthy groups. Box plots show medians, 25th and 75th

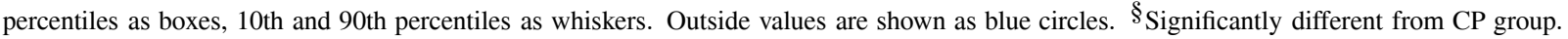
(Kruskal Wallis test, $p<0.05$, Mann-Whitney $\mathrm{U}$ test, $p<0.008$ ).

study has shown that GCF calprotectin total amount is enhanced with the severity of periodontal disease and significantly elevated in $\mathrm{G}-\mathrm{AgP}$ and $\mathrm{CP}$ compared to other study groups. In relation to bone turnover markers, CP group had lower osteocalcin/NTx ratio while $\mathrm{G}-\mathrm{AgP}$, gingivitis and healthy groups had similar ratio. The role of these biomarkers in the pathogenesis of cyclosporine-induced gingival overgrowth was recently investigated by the same study group [39]. Our data has provided the first evidence about the presence of NTx in GCF of patients with different periodontal diseases.

It is known that various sampling protocols are present regarding GCF collection [37,40], still no general agreement was currently present for regarding sampling method. In the present study, paper strips were carefully inserted into the crevice until mild resistance was felt and left there for 30 seconds [37]. Although this technique provides larger GCF volumes compared to superficial intracrevicular technique [40], in accor- 
Table 2

Correlations between calprotectin, osteocalcin, NTX and osteocalcin/NTx and clinical parameters of $\mathrm{G}-\mathrm{AgP}, \mathrm{CP}$, gingivitis and healthy groups

\begin{tabular}{lcccc}
\hline Clinical parameters & Calprotectin & Osteocalcin & NTx & Osteocalcin/NTx \\
\hline PD $(\mathrm{mm})$ & $0.767^{*}$ & $-0.643^{*}$ & 0.067 & $-0.605^{*}$ \\
CAL $(\mathrm{mm})$ & $0.736^{*}$ & $-0.559^{*}$ & 0.078 & $-0.511^{*}$ \\
PBI & $0.577^{*}$ & $-0.406^{*}$ & -0.141 & $-0.330^{*}$ \\
PI & $0.451^{*}$ & $-0.403^{*}$ & -0.030 & $-0.340^{*}$ \\
GCF $(\mu \mathrm{l})$ & $0.401^{*}$ & $-0.370^{*}$ & -0.187 & $-0.273^{*}$ \\
\hline
\end{tabular}

Spearman correlation $\left({ }^{*} p<0.05\right)$. PD: Probing depth, CAL: Clinical attachment loss, PBI: Papillary bleeding index, PI: Plaque index, GCF: Gingival crevicular fluid. G-AgP: Generalized aggressive periodontitis, CP: Chronic periodontitis.

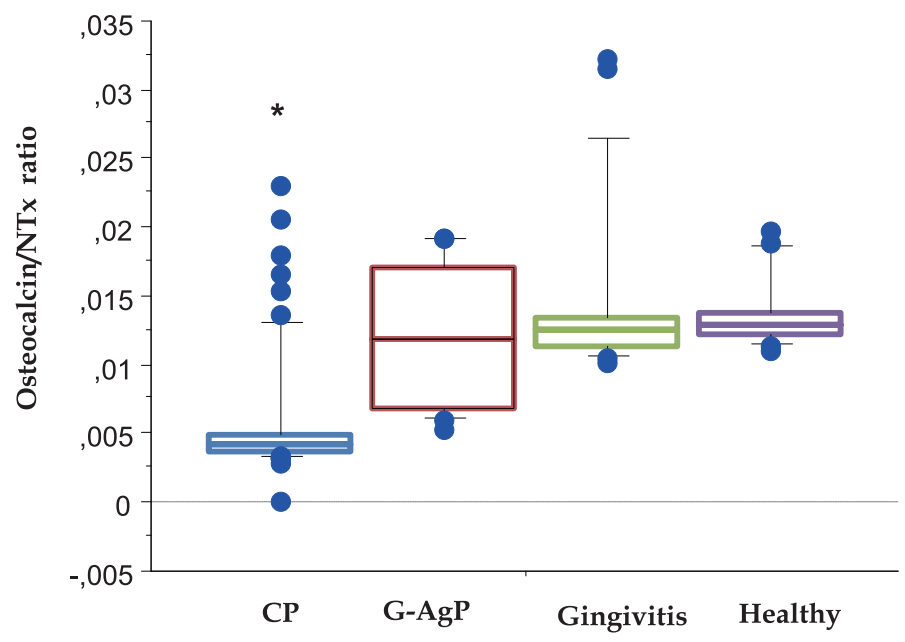

Fig. 4. GCF osteocalcin/NTx ratio of CP, G-AgP, gingivitis and healthy groups. Box plots show medians, 25 th and 75 th percentiles as boxes, 10 th and 90 th percentiles as whiskers. Outside values are shown as blue circles. ${ }^{*}$ Significantly different from G-AgP, gingivitis and healthy groups. (Kruskal Wallis test, $p<0.05$, Mann-Whitney U test, $p<0.008$ ).

dance with the suggestions of others our discussion of the data as well as studying relationship with clinical data was based on the total amount rather than concentration of the data which consider the amount of GCF collected [37,41].

The relationship between GCF calprotectin levels and several other inflammatory markers has been previously investigated and calprotectin levels were shown to be a significant contributor to host defense against infection $[8,9,42,43]$. The present data indicated that GCF calprotectin levels increased as the periodontal disease progresses from health to disease, but similar in G-AgP and CP. Our findings are consistent with the previous data showing the role of calprotectin as an inflammatory marker of periodontal disease $[11,12,42$, 43]. Kaner et al. [44] have demonstrated that GCF calprotectin levels are significantly correlated with the levels of periodontal inflammation and decreased after periodontal therapy in G-AgP patients. Elevated GCF calprotectin levels in diseased groups might be due to the increased activation of inflammatory cells by bac- terial components as well as greater release of intracellular material in gingivitis and periodontitis [45-47]. As a result, calprotectin could contribute to the host inflammatory immune defense against bacteria in periodontal disease $[8,9,48,49]$. In an in vitro study, epithelial calprotectin was shown to promote resistance to Porphyromonas gingivalis invasion and thereby reduce bacterial invasion [8]. Based on the present data we suggest that calprotectin could be a reliable GCF biomarker of gingivitis and periodontitis. On the other hand, similar GCF calprotectin levels in both G-AgP and $\mathrm{CP}$ groups might show that magnitude of the inflammatory component of the host response is not different in these clinically distinct periodontal diseases as has been stated [50].

It is well known that the homeostasis in bone turnover is perturbed during the progression of periodontal disease and leads to the irreversible bone resorption where immune cells and cytokines released play an important role [5]. It is of importance to investigate both bone formation and resorption markers to understand abnor- 
mal bone turnover rate in periodontitis. In the present cross-sectional study, both osteocalcin as a marker of bone formation and NTx as a marker of bone resorption were investigated to have an idea about the change in bone turnover in different periodontal disease, in other words how the levels of these biochemical markers changes in GCF while the disease progress from gingivitis to periodontitis. Osteocalcin/NTx ratio has been suggested to be a marker of bone turnover normalized with respect to resorption [33]. In the present study, this ratio was also calculated in order to evaluate how bone turnover changes in different periodontal diseases. The presence of measurable amount of GCF osteocalcin and NTx in gingivitis and healthy groups might be due to the normal bone homeostasis.

Serum osteocalcin levels increase in several diseases such as osteoporosis where rapid bone turnover are seen and accepted as a valid marker of bone turnover when resorption and formation are coupled [22,51]. It has been previously shown that bone turnover profiles from periodontal bone surfaces and GCF differed from systemic bone turnover profiles [33]. In periodontitis osteocalcin has been suggested to be a marker of bone formation where bone resorption is greater than formation, and GCF osteocalcin levels are more revealing than serum or saliva levels regarding bone turnover in periodontium [52]. On the other hand, there is a controversy about the GCF osteocalcin levels in periodontal diseases. Kunimatsu et al. [53] did not find osteocalcin in GCF of patients with gingivitis, while in periodontitis GCF osteocalcin was positively correlated with clinical parameters. Lee et al. [32] demonstrated similar GCF osteocalcin levels in diseased and healthy sites in patients with CP. Wilson et al. [33] could not detect osteocalcin in GCF of untreated periodontitis patients. On the other hand, Nakashima et al. [54] found elevated osteocalcin total amount in GCF from periodontitis sites compared to those found in healthy and gingivitis sites. In the present study both periodontitis groups (G$\mathrm{AgP}$ and $\mathrm{CP}$ ) had lower osteocalcin levels compared to gingivitis and healthy groups. Furthermore, GCF osteocalcin levels were negatively correlated with periodontal parameters. These findings might point out to the abnormal bone turnover in periodontitis. Moreover, variations in osteocalcin levels among different studies as well as the current study might reflect inability to differentiate between sites undergoing attachment loss and others in a "bone loss arrest" state, where clinical signs of PD ( CAL, increased PPD, bleeding on probing) are present, but no activity in present.

The present data show that NTx, originally identified in urine as a product of osteoclastic bone resorp- tion [21], can also be measured in GCF of patients with different periodontal diseases. $\mathrm{CP}$ and $\mathrm{G}-\mathrm{AgP}$ groups had similar GCF NTx levels with gingivitis and healthy but G-AgP group had lower levels compared to the CP group. The presence of GCF NTx in untreated periodontitis has been previously reported and suggested that NTx may be a useful marker of active periodontal bone loss [33]. Friedman et al. [54] evaluated the NTx levels in GCF and peri-implant crevicular fluid (PCF) and speculated that increased NTx levels may predict extensive bone destruction earlier than the GCF and PCF calprotectin levels. To the best of our knowledge this is the first study investigating GCF NTx levels in different forms of periodontal diseases. Therefore, we were not able to compare our results with others. Increased levels of NTx as a reliable and sensitive biochemical marker of bone resorption have been shown in several systemic diseases associated with bone loss [23, 24,51].

It is known that the quality of the host immuneinflammatory response against bacterial challenge determines the severity and extent of disease. CP and $\mathrm{G}-\mathrm{AgP}$ are different disease entities with different etiology and pathogenesis $[4,56]$. In the present study, GAgP patients had significantly lower GCF NTx levels compared to CP patients. This is an interesting finding of the present study since rapid and severe periodontal destruction occurs in G-AgP compared to $\mathrm{CP}$ [4]. Considering the role of NTx as a resorption marker in bone turnover one could expect higher GCF NTx and lower osteocalcin/NTx ratio in G-AgP. The diversity in bone-specific markers of tissue breakdown in $\mathrm{G}-\mathrm{AgP}$ and $\mathrm{CP}$ groups might indicate high (or abnormal) bone turnover rate in both G-AgP and CP. Alternatively, GCF NTx levels might reflect the catabolic stage of the bone metabolic activity that may be low in G-AgP due to other as yet unidentified factors. As aggressive form of periodontitis seems to have a rapid periodontal destruction as well as higher rate of bone loss compared to the chronic form of periodontitis, whether or not these markers play a role on this rapid progression seen in $\mathrm{G}-\mathrm{AgP}$ deserves further investigation.

In conclusion, our data show that patient groups differ considerably in their capacity to release osteocalcin and NTx as well as calprotectin in GCF. Decreased or unchanged GCF levels of bone turnover markers as well as calprotectin levels in periodontal diseases amend and further extend the understanding of the pathogenesis of periodontal diseases. Our data document for the first time the presence of NTx in GCF of different periodontal diseases. The cross-sectional nature of the 
present study limits its ability to make causal relationship and the findings should be confirmed by longitudinal studies investigating the levels of these biomarkers after periodontal treatment. Additional studies are necessary to clear the role, regulation and function of these molecules in the pathogenesis of periodontal disease as well as if these play a role in distinct forms of periodontitis.

\section{References}

[1] P.N. Madianos, Y.A. Bobetsis and D.F. Kinane, Generation of inflammatory stimuli: how bacteria set up inflammatory responses in the gingiva, J Clin Periodontol 8 (2005), 57-71.

[2] A. Amano, Host-parasite interactions in periodontitis: microbial pathogenicity and innate immunity, Periodontol 200053 (2010), 9-14.

[3] K.S. Kornman, R.C. Page and M.S. Tonetti, The host response to the microbial challenge in periodontitis: assembling the players, Periodontol 200014 (1997), 33-53.

[4] G.C. Armitage and M.P. Cullinan, Comparison of the clinical features of chronic and aggressive, Periodontol $2000 \mathbf{5 3}$ (2010), 12-27.

[5] M. Koide, S. Kinugawa, N. Takahashi and N. Udagawa, Osteoclastic bone resorption induced by innate immune responses, Periodontol 200054 (2010), 235-246.

[6] S. Yui, Y. Nakatani and M. Mikami, Calprotectin (S100A8/ S100A9), an inflammatory protein complex from neutrophils with a broad apoptosis-inducing activity, Biol Pharm Bull 26 (2003), 753-760.

[7] I. Stríz and I. Trebichavský, Calprotectin - a pleiotropic molecule in acute and chronic inflammation, Physiol Res $\mathbf{5 3}$ (2004), 245-253.

[8] K. Nisapakultorn, K.F. Ross and M.C. Herzberg, Calprotectin expression in vitro by oral epithelial cells confers resistance to infection by Porphyromonas gingivalis, Infect Immun 69 (2001), 4242-4247.

[9] K.T Miyasaki, A.L. Bodeau, A.R. Murthy and R.I. Lehrer, In vitro antimicrobial activity of the human neutrophil cytosolic S-100 protein complex, calprotectin, against Capnocytophaga sputigena, J Dent Res 72 (1993), 517-523.

[10] J. Kido, S. Nishikawa, H. Ishida, K. Yamashita, S. Kitamura, K. Kohri and T. Nagata, Identification of calprotectin, a calcium binding leukocyte protein, in human dental calculus matrix, J Periodontal Res 32 (1997), 355-361.

[11] J. Kido, T. Nikato, R. Kido, K. Ohishi, N. Yamauchi, M. Kataoka and T. Nagata, Calprotectin, a leukocyte protein related to inflammation, in gingival crevicular fluid, J Periodontal Res 33 (1998), 434-447.

[12] T. Kojima, E. Andersen, J.C. Sanchez, M.R. Wilkins, D.F. Hochstrasser, W.F. Pralong and G. Cimasoni, Human gingival crevicular fluid contains MRP8 (S100A8) and MRP14 (S100A9), two calcium-binding proteins of the S100 family, J Dent Res 79 (2000), 740-747.

[13] F.T. Lundy, R. Chalk, P.J. Lamey, C. Shaw and G.J. Linden, Identification of MRP-8 (calgranulin A) as a major responsive protein in chronic periodontitis, J Pathol 192 (2000), 540-544.

[14] F.T. Lundy, R. Chalk, P.J. Lamey, C. Shaw and G.J. Linden, Quantitative analysis of MRP-8 in gingival crevicular fluid in periodontal health and disease using microbore HPLC, $J$ Clin Periodontol 28 (2001), 1172-1177.
[15] O. Raska, K. Bernásková and I.JR. Raska, Bone metabolism: a note on the significance of mouse models, Physiol Res $\mathbf{5 8}$ (2009), 459-471.

[16] R.H. Christenson, Biochemical markers of bone metabolism: an overview, Clin Biochem 30 (1997), 573-593.

[17] R. Civitelli, R. Armamento-Villareal and N. Napoli, Bone turnover markers: understanding their value in clinical trials and clinical practice, Osteoporos Int 20 (2009), 843-851.

[18] P.V. Hauschka, J.B. Lian, D.E. Cole and C.M. Gundberg, Osteocalcin and matrix Gla protein: vitamin K-dependent proteins in bone, Physiol Rev 69 (1989), 990-1047.

[19] A.J. Lee, S. Hodges and R.Eastell, Measurement of osteocalcin, Ann Clin Biochem 37 (2000), 432-446.

[20] P.V. Hauschka, Osteocalcin: the vitamin K-dependent Ca2+binding protein of bone matrix, Haemostasis 16 (1986), 258272.

[21] D.A. Hanson, M.A. Weis, A.M. Bollen, S.L. Maslan, F.R. Singer and D.R. Eyre, A specific immunoassay for monitoring human bone resorption: quantitation of type I collagen crosslinked N-telopeptides in urine, J Bone Miner Res 7 (1992), 1251-1258.

[22] D.A. Hanson and D.R. Eyre, Molecular site specificity of pyridinoline and pyrrole cross-links in type I collagen of human bone, J Biol Chem 271 (1996), 26508-26516.

[23] J.D. Clemens, M.V. Herrick, F.R. Singer and D.R. Eyre, Evidence that serum NTx (collagentype I N-telopeptides) can act as an immunochemical marker of bone resorption, Clin Chem 43 (1997), 2058-2063.

[24] J. Iwamoto, T. Takeda and S. Ichimura, Urinary cross-linked $\mathrm{N}$-telopeptides of type I collagen levels in patients with rheumatoid arthritis, Calcif Tissue Int 72 (2003), 491-497.

[25] H. Takayanagi, Inflammatory bone destruction and osteoimmunology, J Periodont Res 40 (2005), 287-293.

[26] D.L. Cochran, Inflammation and bone loss in periodontal disease, J Periodontol 79 (2008), 1569-1576.

[27] S.H. Lee, T.S. Kim, Y. Choi and J. Lorenzo, Osteoimmunology: cytokines and the skeletal system, BMB reports 31 (2008), 495-510.

[28] J.T. Talonpoika and M.M. Hämäläinen, Type I collagen carboxyterminal telopeptide in human gingival crevicular fluid in different clinical conditions and after periodontal treatment, $J$ Clin Periodontol 21 (1994), 320-326.

[29] J. Kido, T. Nakamura, Y. Asahara, T. Sawa, K. Kohri and T. Nagata, Osteopontin in gingival crevicular fluid, $J$ Periodont Res 36 (2001), 328-333.

[30] C.G. Sharma and A.R. Pradeep, Gingival crevicular fluid osteopontin levels in periodontal health and disease, J Periodontol 77 (2006), 1674-1680.

[31] K. Nakashima, C. Giannopoulou, E. Andersen, N. Roehrich, P. Brochut, B. Dubrez and G. Cimasoni, A longitudinal study of various crevicular fluid components as markers of periodontal disease activity, J Clin Periodontol 23 (1996), 832-838.

[32] A.J. Lee, T.F. Walsh, S.J. Hodges and A. Rawlinson, Gingival crevicular fluid osteocalcin in adult periodontitis, J Clin Periodontol 26 (1999), 252-256.

[33] A.N. Wilson, M.J. Schmid, D.B. Marx and R.A. Reinhardt, Bone turnover markers in serum and periodontal microenvironments, J Periodont Res 38 (2003), 355-361.

[34] G.C. Armitage, Development of a classification system for periodontal diseases and conditions, Annal Periodontol 4 (1999), $1-7$.

[35] U.P. Saxer and H.R. Mühlemann, Motivation and instruction (in German), SSO Schweiz Monatsschr Zahnheilkd 85 (1975), 905-919. 
[36] G.A. Quigley and J.W. Hein, Comparative cleansing efficiency of manual and power brushing, J Am Dent Assoc 65 (1962), 26-29.

[37] I.B. Lamster, L.J. Hartley and R.L. Oshrain, Evaluation and modification of spectrophotometric procedures for analysis of lactate dehydrogenase, beta-glucuronidase and arylsulphatase in human gingival crevicular fluid collected with filter-paper strips, Arch Oral Biol 30 (1985), 235-242.

[38] G. Cimasoni and S. Karger, Method of collection, Crevicular Fluid Updated (1983), 29-36.

[39] S. Becerik, A. Gürkan, B. Afacan, VÖ. Öztürk, H. Atmaca, H. Töz, G. Atilla and G. Emingil, Gingival Crevicular Fluid Osteocalcin, NTx and Calprotectin Levels in Cyclosporine a Induced Gingival Overgrowth, J Periodontol (22 Feb 2011), [Epub ahead of print].

[40] H. Löe and P. Holm-Pedersen, Absence and presence of fluid from normal and inflamed gingivae, Periodontics 149 (1965), 171-177.

[41] I.B. Lamster, R.L. Oshrain and J.M. Gordon, Enzyme activity in human gingival crevicular fluid: Considerations in data reporting based on analysis of individual crevicular sites, $J$ Clin Periodontol 13 (1986), 799-804.

[42] J. Kido, T. Nakamura, R. Kido, K. Ohishi, N. Yamauchi, M. Kataoka and T. Nagata, Calprotectin in gingival crevicular fluid correlates with clinical and biochemical markers of periodontal disease, J Clin Periodontol 26 (1999), 653-657.

[43] T. Nakamura, J. Kido, R. Kido, K. Ohishi, N. Yamauchi, M. Kataoka and T. Nagata, The association of calprotectin level in gingival crevicular fluid with gingival index and the activities of collagenase and aspartate aminotransferase in adult periodontitis patients, J Periodontol 71 (2000), 361-367.

[44] D. Kaner, J.P. Bernimoulin, B.M. Kleber, W.R. Heizmann and A. Friedmann, Gingival crevicular fluid levels of calprotectin and myeloperoxidase during therapy for generalized aggressive periodontitis, J Periodont Res 41 (2006), 132-139.

[45] C.M. Champagne, W. Buchanan, M.S. Reddy, J.S. Preisser, J.D. Beck and S. Offenbacher, Potential for gingival crevice fluid measures as predictors of risk for periodontal diseases, Periodontol 200031 (2003), 167-180.

[46] J. Kido, R. Kido, M. Suryono, M. Kataoka, M.K. Fagerhol and T. Nagata, Calprotectin release from human neutrophils is induced by Porphyromonas gingivalis lipopolysaccharide via the CD-14-Toll-like receptor-nuclear factor $\kappa \mathrm{B}$ pathway, $J$ Periodont Res 38 (2003), 557-563.

[47] J. Kido, R. Kido, M. Suryono, M. Kataoka, M.K. Fagerhol and T. Nagata, Induction of calprotectin release by Porphyromonas gingivalis lipopolysaccharide in human neutrophils, Oral Microbiol Immunol 19 (2004), 182-187.

[48] M. Suryono, J. Kido, N. Hayashi, M. Kataoka and T. Nagata, Effect of Porphyromonas gingivalis lipopolysaccharide, tumor necrosis factor-alpha, and interleukin-1beta on calprotectin release in human monocytes, J Periodontol 74 (2003), 17191724.

[49] M. Suryano, J. Kido, N. Hayashi, M. Kataoka and T. Nagata, Calprotectin expression in human monocytes: induction by Porphyromonas gingivalis lipopolysaccharide, tumor necrosis factor-alpha, and interleukin-1beta, J Periodontol 76 (2005), 437-442.

[50] R. Buchmann, A. Hasilik, T.E. Van Dyke and D.E. Lange, Amplified crevicular leukocyte activity in aggressive periodontal disease, J Dent Res 81 (2002), 716-721.

[51] P.D. Delmas, R. Eastell, P. Garnero, M.J. Seibel and J. Stepan, The use of biochemical markers of bone turnover in osteoporosis, Osteoporos Int 6 (2000), 2-17.

[52] R.A. Reinhardt, V.J. Sanderfer, T.A. Meinberg, P. Nummikoski, H.M. Lee and D.B. Marx, Local biochemical markers of bone turnover: relationship to subsequent density of healing alveolar bone defects, J Clin Periodontol 31 (2004), 223-228.

[53] K. Kunimatsu, S. Mataki, H. Tanaka, N. Mine, M. Kiyoki, K. Hosoda, Y. Kato and I. Kato, A cross-sectional study on osteocalcin levels ingingival crevicular fluid from periodontal patients, J Periodontol 64 (1993), 865-869.

[54] K. Nakashima, N. Roehrich and G. Cimasoni, Osteocalcin, prostaglandin E2 and alkaline phosphatase in gingival crevicular fluid: Their relations to periodontal status, J Clin Periodontol 21 (1994), 327-333.

[55] A. Friedmann, M. Friedrichs, D. Kaner, B.M. Kleber and J.P. Bernimoulin, Calprotectin and cross-linked N-terminal telopeptides in peri-implant and gingival crevicular fluid, Clinical oral implants research 17 (2006), 527-532.

[56] P.J. Ford, J. Gamonal and G.J. Seymour, Immunological differences and similarities between chronic periodontitis and aggressive periodontitis, Periodontol 200053 (2010), 111-123. 


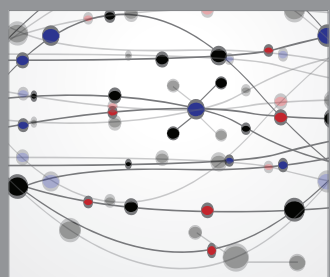

The Scientific World Journal
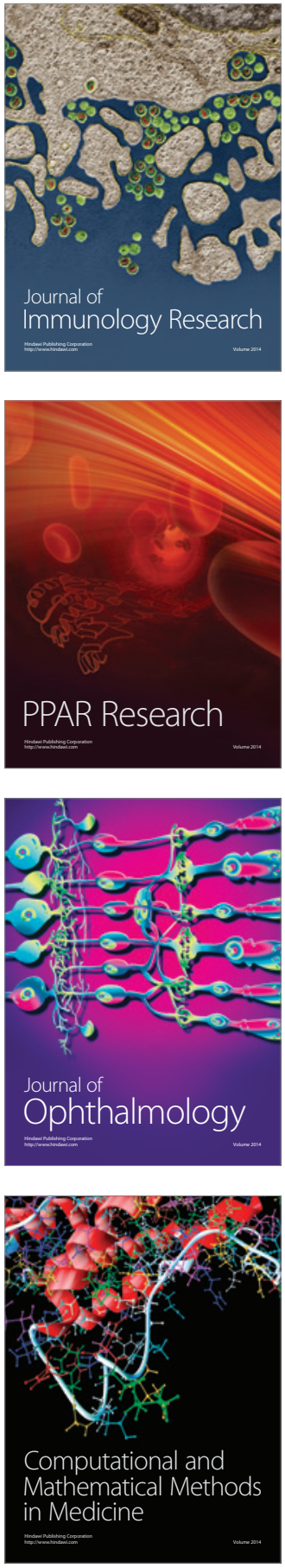

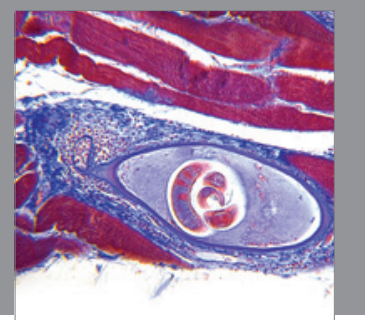

Gastroenterology

Research and Practice
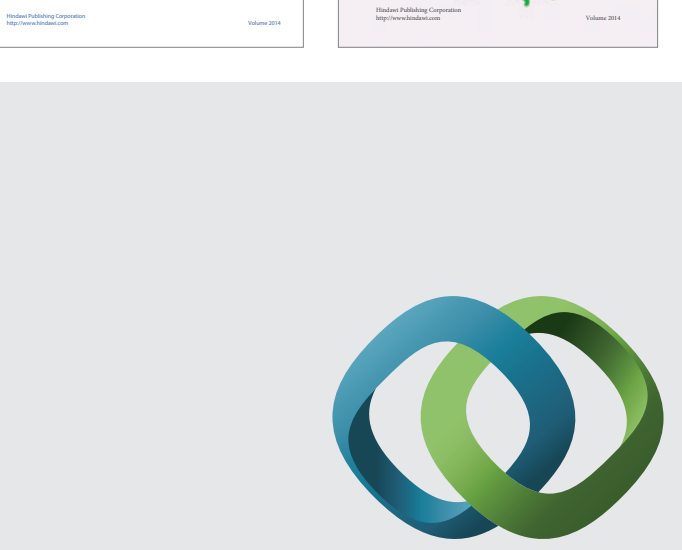

\section{Hindawi}

Submit your manuscripts at

http://www.hindawi.com
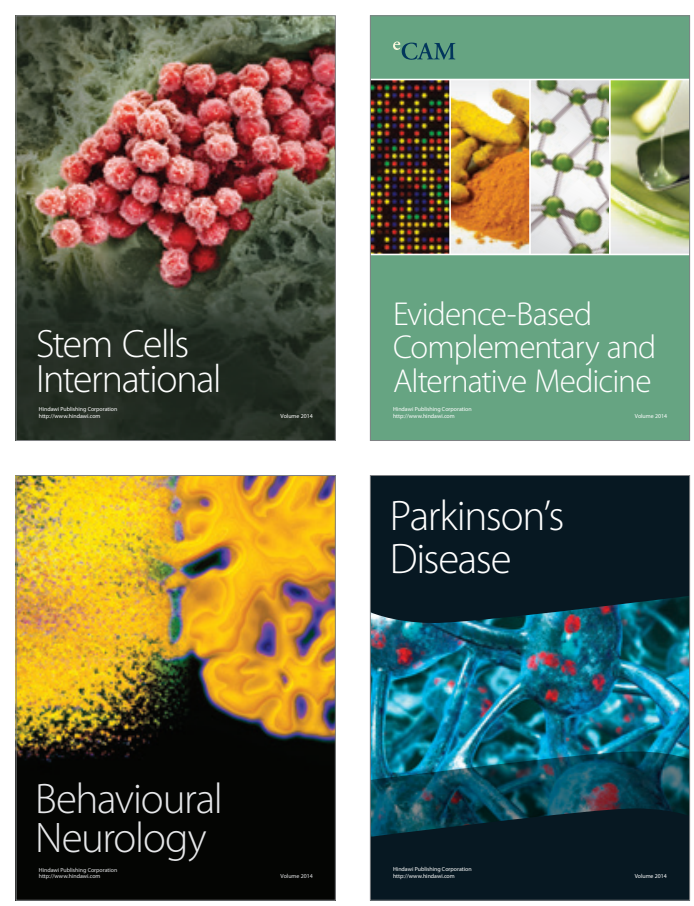

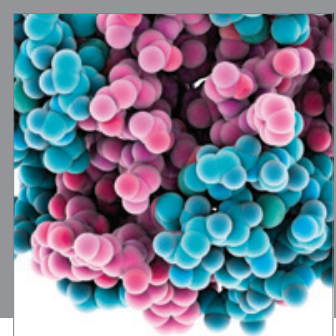

Journal of
Diabetes Research

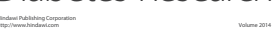

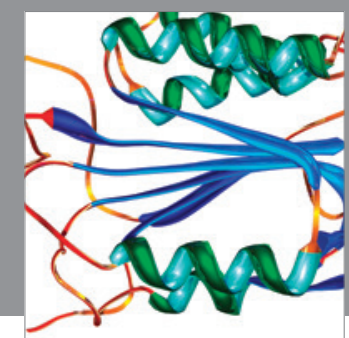

Disease Markers
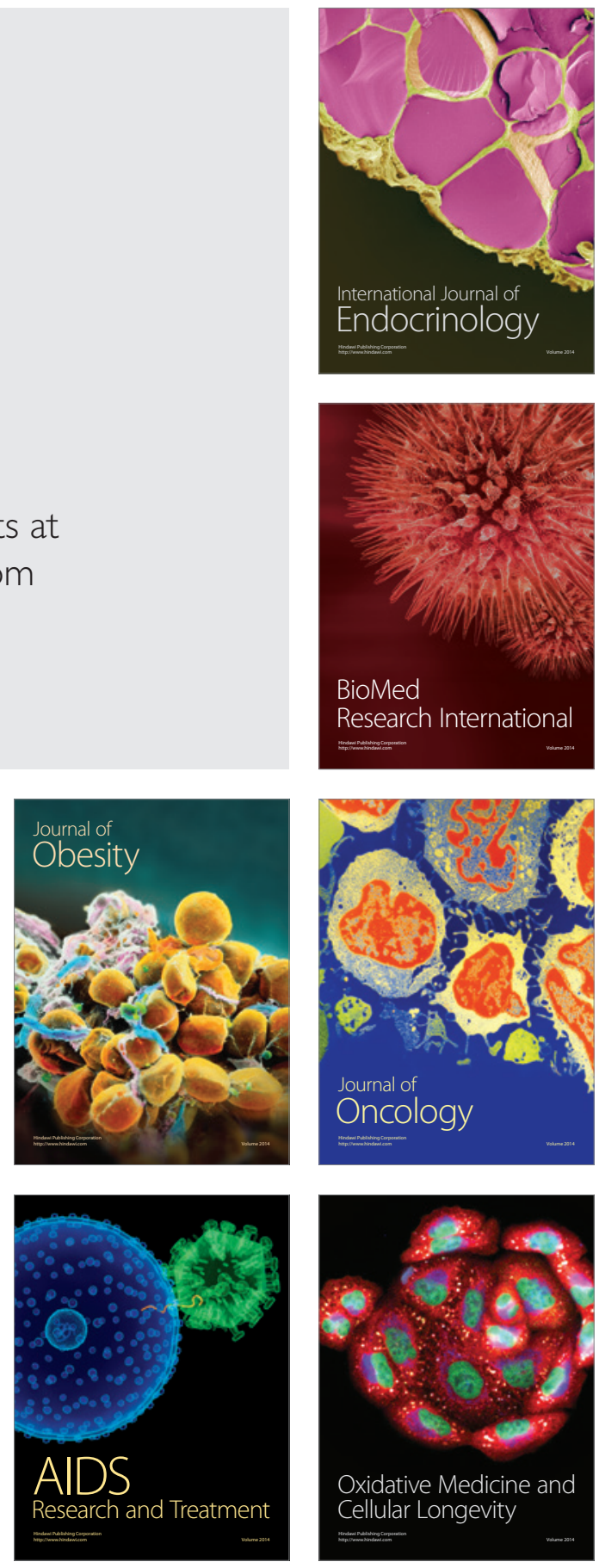